Linköping studies in science and technology

Licentiate Thesis. No. 1825

\title{
Optimisation of Off-Road Transport Missions
}

Jörgen Albrektsson

Department of Electrical Engineering

Linköping University, SE-581 33 Linköping, Sweden

Linköping 2018 
Linköping studies in science and technology

Licentiate Thesis. No. 1825

This is a Swedish Licentiate's Thesis.

Swedish postgraduate education leads to a Doctor's degree and/or a Licentiate's degree.

A Doctor's degree comprises 240 ECTS credits (4 years of full-time studies).

A Licentiate's degree comprises 120 ECTS credits,

of which at least 60 ECTS credits constitute a Licentiate's thesis.

Jörgen Albrektsson

jorgen.albrektsson@volvo.com

www.vehicular.isy.liu.se

Division of Vehicular Systems

Department of Electrical Engineering

Linköping University

SE-581 33 Linköping, Sweden

Copyright (C) 2018 Jörgen Albrektsson, unless otherwise noted.

All rights reserved.

Albrektsson, Jörgen

Optimisation of Off-Road Transport Missions

ISBN 978-91-7685-168-5

ISSN 0280-7971

Typeset with $\mathrm{IAT}_{\mathrm{EX}} 2_{\varepsilon}$

Printed by LiU-Tryck, Linköping, Sweden 2018 
To Sara and Klara 



\section{Abstract}

Mines, construction sites, road construction and quarries are examples of applications where construction equipment are used. In a production chain consisting of several construction machines working together, the work needs to be optimised and coordinated to achieve an environmental friendly, energy efficient and productive production. Recent rapid development within positioning services, telematics and human machine interfaces (HMI) opens up for control of individual machines and optimisation of transport missions where several construction machines co-operate.

The production chain on a work site can be split up in different sub-tasks of which some can be transport missions. Taking off in a transport mission where one wheel loader ("loader" hereinafter) and two articulated haulers ("haulers" hereinafter) co-operate to transport material at a set production rate [ton/h], a method for fuel optimal control is developed. On the mission level, optimal cycle times for individual sub-tasks such as wheel loader loading, hauler transport and hauler return, are established through the usage of Pareto fronts.

The haulers Pareto fronts are built through the development of a Dynamic Programming (DP) algorithm that trades fuel consumption versus cycle time for a road stretch by means of a time penalty constant. Through varying the time penalty constant $n$ number of times, discrete fuel consumption - cycle time values can be achieved, forming the Pareto front. At a later stage, the same DP algorithm is used to generate fuel optimal vehicle speed and gear trajectories that are used as control signals for the haulers. Input to the DP algorithm is the distance to be travelled, road inclination, rolling resistance coefficient and a max speed limit to avoid unrealistic optimisation results.

Thus, a method to describe the road and detect the road related data is needed to enable the optimisation. A map module is built utilising an extended Kalman Filter, Rauch-Tung-Striebel smoother and sensor fusion to merge data and estimate parameters not observable by sensors. The map module uses a model of the vehicle, sensor signals from a GPS or GNSS sensor and machine sensors to establish a map of the road.

The wheel loader Pareto front is based on data developed in previous research combined with Volvo in-house data. The developed optimisation algorithms are implemented on a PC and in an interactive computer tablet based system. A human machine interface is created for the tablet, guiding the operators to follow the optimal control signals, which is speed for the haulers and cycle time for the loader. To evaluate the performance of the system it is tested in real working conditions.

The contributions develop algorithms, set up a demo mission control system and carry out experiments. Altogether rendering in a platform that can be used as a base for a future design of an off-road transport mission control system. 



\section{ACKNOWLEDGMENTS}

It's today's fashion to talk about journeys but to me the time spent towards the completion of this thesis more resembles a bike race. A very enthusiastic start, steep hills with hard studies and disappointing results, tedious flats of writing, easy going down slopes as code comes into place and results starts to show, and then, the obvious race towards the finish line.

I would like to thank the team's main coach: my supervisor associate Professor Jan Åslund, for support and for steering the bike in the right direction. I would also like to thank Rikard Mäki and Anders Fröberg for putting me on the start line, the Fordonsystem team at Linköping University for interesting and fun discussions and Andreas Myklebust for the introduction to being a PhD student and the world of LaTex.

I'm grateful to my second supervisor Professor Lars Eriksson, FS colleagues Vaheed Nezhadali and Xavier Llamas Comellas, to former and present Volvo CE colleagues Peter Wallin, Erik Uhlin and Johan Sjöberg and my family for giving the necessary support to keep the pedals moving.

The research is jointly financed by Volvo Construction Equipment and the "Efficient and connected transport system" program by FFI (Fordonstrategisk Forskning och Innovation).

Finally, a huge hug to Sara and Klara, the inspiration in my life!

Jörgen Albrektsson

Linköping, October 2018 

Contents

1 Introduction 1

1.1 An Off-Road Transport Mission . . . . . . . . . . . . . . . . 1

1.2 Scope, Objective and Positioning of the Work . . . . . . . . . . 4

1.3 Contributions . . . . . . . . . . . . . . . . . 4

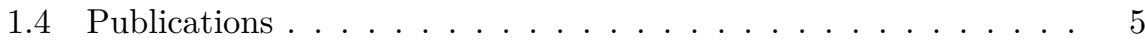

$\begin{array}{ll}\text { Publications } & 9\end{array}$

A Road Estimation and Fuel Optimal Control of an Off-Road Ve$\begin{array}{lr}\text { hicle } & 11\end{array}$

1 INTRODUCTION . . . . . . . . . . . . . . . . . . . 14

2 VEHICLE MODEL . . . . . . . . . . . . . . . . . 14

2.1 External Forces Acting on the Hauler . . . . . . . . . 15

2.2 Drivetrain Model . . . . . . . . . . . . . . . . . . 15

2.3 Complete Vehicle Model . . . . . . . . . . . . . . . 19

2.4 Model Alteration for Optimisation . . . . . . . . . . . . 19

3 ESTIMATION OF ROAD INCLINATION AND ROLLING RESISTANCE . . . . . . . . . . . . . . . 20

3.1 Map Building Process . . . . . . . . . . . . . . . 20

3.2 Sensor and Data Fusion . . . . . . . . . . . . . 21

4 OPTIMAL CONTROL OF AN ARTICULATED HAULER . . . 25

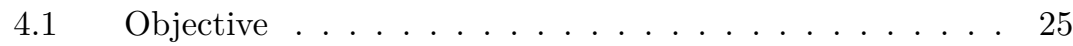

4.2 Dynamic Programming . . . . . . . . . . . . 25

4.3 Dynamic Model . . . . . . . . . . . . . . . 26

4.4 Discretisation . . . . . . . . . . . . . 26

4.5 Cost Function . . . . . . . . . . . . . . . . . 27

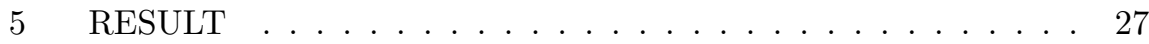

$6 \quad$ CONCLUSION . . . . . . . . . . . . . . . 31 
B Fuel Optimal Control of an Articulated Hauler Utilising a Human Machine Interface

1 Introduction . . . . . . . . . . . . . . . . . . . 38

2 Theoretical Basis . . . . . . . . . . . . . . . . . . 38

$2.1 \quad$ Vehicle Model . . . . . . . . . . . . . . . . . . . . . . . . 39

2.2 Map Module . . . . . . . . . . . . . . . . . . . 40

2.3 Optimisation Module . . . . . . . . . . . . . . . . 44

3 Human Machine Interface . . . . . . . . . . . . . . . . . . . . 46

3.1 Hardware . . . . . . . . . . . . . . . . . . . . . 46

3.2 Implementation . . . . . . . . . . . . . . . 46

4 Machine Tests . . . . . . . . . . . . . . . . . . . 48

$5 \quad$ Result . . . . . . . . . . . . . . . . . 50

5.1 Discussion . . . . . . . . . . . . . . . . 50

6 Conclusion .................. 56

C Fuel Optimal Control of an Off-Road Transport Mission $\quad 59$

1 Introduction . . . . . . . . . . . . . 62

2 Transport Mission Optimisation . . . . . . . . . . . . . 62

$2.1 \quad$ Articulated Hauler Pareto Front . . . . . . . . . . . 63

2.2 Wheel Loader Pareto Front . . . . . . . . . . . . . . . 64

2.3 Optimisation of Transport Mission . . . . . . . . . . 65

3 Algorithm implementation . . . . . . . . . . . . . . 66

$3.1 \quad$ Human Machine Interface . . . . . . . . . . . . . . 67

4 Machine Tests . . . . . . . . . . . . . . . . . . 67

$4.1 \quad$ Baseline . . . . . . . . . . . . . . . . . . . . 69

$4.2 \quad$ Test case . . . . . . . . . . . . . . . . . . . . . 69

$5 \quad$ Result . . . . . . . . . . . . . . . . . . . 69

5.1 Test case 1: Transport Without Meeting . . . . . . . 70

5.2 Test case 2: Transport With Meeting . . . . . . . . . 71

5.3 Discussion . . . . . . . . . . . . . . . . . . . . . 72

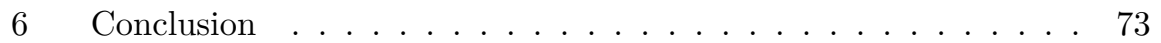




\section{Chapter 1}

\section{Introduction}

Taking off in the 70'ies oil crises, minimising energy consumption in general, and for the transport sector fuel consumption especially, has been a high priority to society. Later on the environmental impact of utilising internal combustion engines (ICE) has put even higher focus on minimising fuel consumption from transports. Additionally, there is the obvious benefit of saving money for the end customer, all adding to the need to maximise fuel efficiency for all kinds of transports.

Construction equipment are used in a wast number of applications in e.g. mines, at construction sites, road constructions and others. One example of application is quarries where the rock is blasted into pieces and subsequently crushed in crushers to produce grit in different size. A schematic picture of a quarry, with different construction equipment in operation, is shown in Figure 1.1. The complete production chain in the quarry can be split into sub tasks. Many of these sub tasks are carried out by a combination of vehicles, e.g. the transport of shot rock from the point of excavation to the crusher, see step 9 in Figure 1.1 where a wheel loader, articulated haulers and trucks are used. In a production chain consisting of several construction machines working together, the work needs to be optimised and coordinated to achieve an energy efficient, environmental friendly and productive transport chain.

\subsection{An Off-Road Transport Mission}

The work at hand aim to develop a framework for fuel optimal control of a transport mission conducted by multiple co-operating construction machines. A transport mission conducted by a wheel loader and two articulated haulers working towards a fixed production rate target (e.g. set by a crusher), is used as 


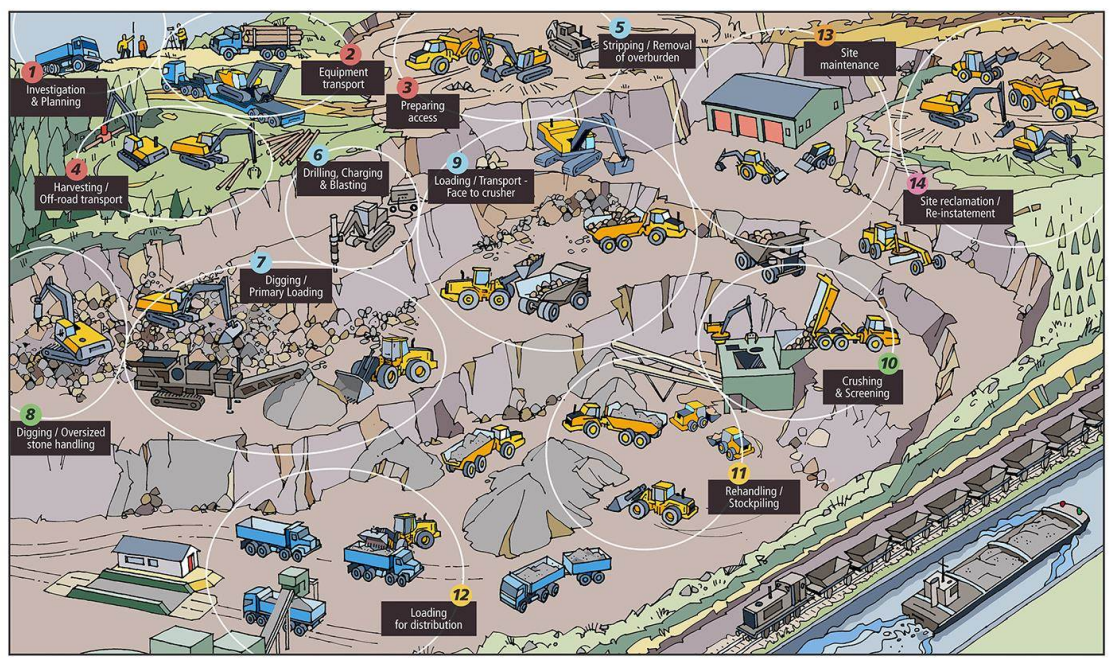

Figure 1.1: Construction equipment working in a quarry. Picture by Volvo CE $\mathrm{AB}$

base for the development and for the subsequent tests of the system. However, the developed methods can be extended to include more machines and the machines can be substituted for others if desired.

On road heavy duty truck transports have attracted high attention in the research community. Research has been performed in e.g. logistics, fleet optimisation and fuel optimal control. Compared to an on-road transport mission the example transport mission differs in some vital aspects. Off-road the distance of the transport is usually much shorter, no official statistics was found but a distance less than $2 \mathrm{~km}$ and $6 \mathrm{~min}$. travel time can be considered normal. E.g. in Europe, $58.7 \%$ of the road transports are between 150 and $1000 \mathrm{~km}$ (Eurostat, 2017). With the shorter distance the possibility to handle data of the complete road after each finished run emerges, unlike longer distances where this may be infeasible due to computational burden. The shorter roads makes it possible to store sensor data and to make the optimisation for the complete road stretch.

Off-road the estimation of road characteristics is in some respects more complicated than on-road. Parameters such as the actual position of the road and road surface changes much more often making it necessary to frequently update the road data. On-road databases and maps available on the internet is easier to use since the routing does not change, the surface is usually consistent (asphalt) and the impact of hills and valleys has been diminished during the construction of the road. For the optimisation it is important to have good knowledge of the road inclination and where rapid changes in inclination, such as hills and valleys, are positioned, thus a higher degree of discretisation of the road is needed off-road. 
Off-road transports often have a direct or indirect production rate target, i.e. the transported amount of mass per time unit [ton/h]. If e.g. a crusher is part of the transport mission, the crusher set a demand on the production rate. The production becomes inefficient and with poor quality if the crushers are not fed with enough material. At the same time, the production will be equally inefficient if the material transport is made at excessive speed, resulting in queuing machines at loading point, meeting points and at the crusher. One can imagine numerous other applications where there is a similar transport need with a more or less fixed production rate. One example could be a road construction site where the material from a hill is used to fill out a small valley to create an even road stretch. Often the volume of the material to be moved is known in advance and the time schedule of the construction work sets the production rate. The existence of a target production rate opens up for scheduling of tasks to avoid excessive speeds rendering in inefficient processes. In (Rylander and Axelsson, 2013) up to $33 \%$ machine waiting time in a similar process is reported, indicating a large potential to increase fuel efficiency through using the waiting time to decrease the speed of the vehicles.

As within road transports the fuel consumption differs widely between skilled and unskilled drivers. For passenger cars a $10 \%$ fuel efficiency increase, if driving behaviour is changed, are considered possible on the fleet level (Barkenbus, 2010). Off-road the difference can be even larger, in (Frank et al., 2012) a difference in fuel efficiency of $200 \%$ is reported between skilled/unskilled wheel loader operators.

Eco driving schools and operator training are efficient ways to mitigate the fuel efficiency loss from bad driver behaviour but the effect from the training have been reported to fade after a while (Beusen et al., 2009). However, an operator assist system (Van der Voort et al., 2001) may promote ECO driving over a longer time period.

Recent rapid development within positioning services (GSM localisation, GPS, GNSS), vehicle-to-everything (V2X) communication and human machine interfaces are other enablers for invoking optimal control in an operator assist system that covers more than a single machine (Rylander, 2014). V2X communication can be used to assist the operator with knowledge about the other machines activities on the work site and the information can be used to adjust the control signals if for some reason the optimal schedule of the transport mission could not be followed. Combining optimal control with the knowledge of other machines activities, a powerful operator support system can be built. 


\subsection{Scope, Objective And Positioning of the WORK}

The objective of the research conducted is to reduce the environmental impact of transport missions performed by construction equipment through minimising fuel consumption. This will be achieved through the development of a method to optimise and control the individual vehicles and the interaction between the vehicles. The focus of the work at hand is on a transport mission consisting of one wheel loader and two articulated haulers, forming a common example of a sub task in a production chain where multiple vehicles are part of the complete transport.

The main research question is how optimal control can improve the productivity and energy efficiency in an off-road transport mission. Additional research questions are whether the developed control algorithms can be adequately implemented in standalone prototype ECU units and if the generated control inputs can be efficiently communicated to the operator through a human machine interface (HMI).

Previous research such as (Sarata et al., 2005), (Nezhadali, 2016) and (Fu and Bortolin, 2012) examines how the individual machine are to be operated as efficient as possible. Other research focus on the site level and examines the potential of optimising the production chain (Kannan et al., 1997) and (Fu, 2017). The work at hand positions itself somewhere in between while control of individual machines are used to optimally control transport missions with multiple machines but where the full site is not considered.

\subsection{CONTRibutions}

A framework for fuel optimal control an off-road transport mission is successively formed by paper A to C. Their main contributions are summarized below.

\section{PAPER A}

The main contribution in Paper A is a method to create a Pareto front of fuel consumption versus cycle time for a transport conducted by an Articulated Hauler. A dynamic programming (DP) algorithm is used to determine a discrete set of minimum fuel consumption vs. cycle time points on which the Pareto front is built. The developed DP algorithm can also be set to generate fuel optimal vehicle speed and gear trajectories that controls the haulers travel. A condition for the optimisation of the hauler transport is knowledge of road related parameters such as distance, inclination and rolling resistance. While the energy consumption stemming from rolling resistance is comparably high in an off-road transport it is of interest to estimate the rolling resistance and utilise the knowledge in the optimisation. Paper A includes a method to generate a 
map with latitude and longitude as identification points to which the estimation of the described road related parameters are appended.

\section{PAPER B}

In Paper B the algorithms developed in Paper A are implemented in the Volvo Co-Pilot system. In the centre of the Volvo Co-Pilot system there is a tablet like interactive display to which a GNSS smart receiver can be connected. The system is also able to read sensor signals from the machine via a computer area network $(\mathrm{CAN})$. Around the algorithms a human machine interface is created. The HMI instructs the machine operator to follow the optimal control signal, in this case vehicle speed. The system is tested in real working conditions and when compared to a base line where a fixed speed target is used, a small fuel efficiency gain can be found.

\section{PAPER C}

A novel method for fuel optimal control of an off-road transport mission is developed in paper C. The method utilises Pareto fronts to set the optimal cycle time for the different sub-tasks in the transport mission. The targeted transport mission involves a wheel loader and two articulated haulers. After the optimal cycle times have been established vehicle trajectories are calculated for the hauler sub-tasks. The control signals, cycle time and number of loading cycles for the wheel loader and vehicle speed for the articulated hauler is communicated to the operator through a newly developed HMI for the wheel loader and through the HMI developed in Paper B for the hauler.

\subsection{Publications}

In the research work leading to this Licentiate thesis, the author has published the following conference and journal papers.

- Jörgen Albrektsson and Jan Åslund. Road Estimation and Fuel Optimal Control of an Off-Road Vehicle. Proceedings of the 3rd International Conference on Vehicle Technology and Intelligent Transport Systems, Porto, Portugal, 2017.

(Paper A)

- Jörgen Albrektsson and Jan Åslund. Fuel Optimal Control of an Articulated Hauler Utilising a Human Machine Interface. Smart Cities, Green Technologies, and Intelligent Transport Systems. Springer, Cham., 2018 (Paper B) 
- Jörgen Albrektsson and Jan Åslund. Fuel Optimal Control of an Off-Road Transport Mission. Proceedings of 2018 IEEE International Conference on Industrial Technology (ICIT) Lyon, France, 2018.

(Paper C) 


\section{Bibliography}

Jack N Barkenbus. Eco-driving: An overlooked climate change initiative. Energy Policy, 38(2):762-769, 2010.

Bart Beusen, Steven Broekx, Tobias Denys, Carolien Beckx, Bart Degraeuwe, Maarten Gijsbers, Kristof Scheepers, Leen Govaerts, Rudi Torfs, and Luc Int Panis. Using on-board logging devices to study the longer-term impact of an eco-driving course. Transportation research part D: transport and environment, 14(7):514-520, 2009.

Eurostat. Road freight transport statistics, 2017. URL http: //ec.europa.eu/eurostat/statistics-explained/index.php/Road_ freight_transport_statistics.

Bobbie Frank, Lennart Skogh, and Mats Alaküla. On wheel loader fuel efficiency difference due to operator behaviour distribution. In 2nd International Commercial Vehicle Technology Symposium, CVT, 2012.

Jiali Fu. Evaluating and Improving the Transport Efficiency of Logistics Operations. PhD thesis, KTH, Transport Planning, Economics and Engineering, 2017. QC 20170323.

Jiali Fu and Gianantonio Bortolin. Gear shift optimization for off-road construction vehicles. In Procedia - social and behavioral science, volume 54. SCIENCEDIRECT, 2012.

Govindan Kannan, Julio C Martinez, and Michael C Vorster. A framework for incorporating dynamic strategies in earth-moving simulations. In Proceedings of the 29th conference on Winter simulation, pages 1119-1126. IEEE Computer Society, 1997.

Vaheed Nezhadali. Modeling and Optimal Control of Heavy-Duty Powertrains. PhD thesis, Linköping University, 2016.

David Rylander. Productivity improvements in construction site operations through lean thinking and wireless real-time control. PhD thesis, Mälardalen University, 2014. 
David Rylander and Jakob Axelsson. Lean method to identify improvements for operation control at quarry sites. In 30th International Symposium on Automation and Robotics in Construction (ISARC 2013), August 11 to 15, 2013, Montréal, Canada, 2013.

Shigeru Sarata, Yossewee Weeramhaeng, and Takashi Tsubouchi. Planning of scooping position and approach path for loading operation by wheel loader. In Proceedings of the International Symposium on Automation and Robotics in Construction, Ferrara, Italy, 2005.

Mascha Van der Voort, Mark S Dougherty, and Martin van Maarseveen. A prototype fuel-efficiency support tool. Transportation Research Part C: Emerging Technologies, 9(4):279-296, 2001. 


\section{Paper A}

\section{Road Estimation and Fuel Optimal Control of an Off-Road Vehicle*}

\footnotetext{
*This is a formatted version of "Road Estimation and Fuel Optimal Control of an Off-Road Vehicle" by Jörgen Albrektsson and Jan Åslund. The original version was published in Proceedings of the 3rd International Conference on Vehicle Technology and Intelligent Transport Systems - Volume 1, Porto, Portugal, $201 \%$. (C) SCITEPRESS 2017. Reproduced with the permission of SCITEPRESS. The original version was published in https://www.scitepress.org, and can be found using the Digital Object Identifier (DOI): 10.5220/0006247200580067. The formatting is restricted to changing the article into a single column format and adjusting the reference style.
} 


\section{Paper B}

\section{Fuel Optimal Control of an Articulated Hauler Utilising a Human Machine Interface}

\footnotetext{
${ }^{\star}$ This is a formatted version of "Fuel Optimal Control of an Articulated Hauler Utilising a Human Machine Interface" by Jörgen Albrektsson and Jan Åslund. In Smart Cities, Green Technologies, and Intelligent Transport Systems. Springer Cham., 2018. The formatting is restricted to adjusting the reference style.
} 


\section{Paper C}

\section{Fuel Optimal Control of an Off-Road Transport Mission}

\footnotetext{
${ }^{\star}$ This is a formatted version of "Fuel Optimal Control of an Off-Road Transport Mission" by Jörgen Albrektsson and Jan Åslund. The original version was published in 2018 IEEE International Conference on Industrial Technology (ICIT) Lyon, France, 2018, pp. 175-180. (C) IEEE 2018. Reproduced with the permission of IEEE. The original version was published in https://ieeexplore.ieee.org, and can be found using the Digital Object Identifier (DOI): $10.1109 /$ ICIT.2018.8352172. The formatting is restricted to changing the article into a single column format and adjusting the reference style.
} 


\section{Papers}

The papers associated with this thesis have been removed for copyright reasons. For more details about these see:

http://urn.kb.se/resolve?urn=urn:nbn:se:liu:diva-152787 